\title{
The Newhouse Set Has a Positive Hausdorff Dimension
}

\author{
Xiao-Jing Wang* \\ Faculté des Sciences, Université Libre de Bruxelles, Campus Plaine, c.p. 231, \\ Blvd du Triomphe, B-1050 Bruxelles, Belgium
}

\begin{abstract}
The Newhouse phenomenon of infinitely many coexisting periodic attractors is studied in its simplest form. One shows that the corresponding parameter set (the Newhouse set) $J_{N}$ has a strictly positive Hausdorff dimension. This result is stronger than that of Tedeschini-Lalli and Yorke [Commun. Math. Phys. 106, 635 (1986)] concerning the Lebesgue measure of the Newhouse set; and is complementary to our knowledge on the topological properties of $J_{N}$, namely it is a residual set, hence uncountable and everywhere dense in a parameter interval.
\end{abstract}

\section{Introduction}

It is nowadays well known, and much discussed in the literature, that in a multidimensional dynamical system, an infinite number of periodic attractors may coexist in a bounded region of the phase space. This possibility was recognized largely due to the work by Newhouse $(1974,1979)$. While studying the homoclinic tangency to a periodic saddle point in one-parameter families of planar diffeomorphisms, this author proved that the system near the tangency can possess an invariant basic set of chaotic nature, to which secondary homoclinic tangencies persist for an open set of parameter values. As a consequence, there is a residual parameter set (the Newhouse set), for which infinitely many sinks of arbitrarily long period coexist in the system.

One may naturally ask how typically does this phenomenon occur, or how generic is the Newhouse parameter set? We note immediately that there are two distinct basic notions of genericity for a set of real numbers: measure and category (Oxtoby, 1980). Being residual, the Newhouse set is generic in the topological sense of belonging to Baire's second category. It is not countable, and everywhere dense in an interval. The measure properties have been discussed by Tedeschini-Lalli and

\footnotetext{
* Current address: Mathematical Research Branch, NIDDK, Bldg 31, Room 4B-54, National Institutes of Health, Bethesda, MD 20892, USA
} 
Fig. 1

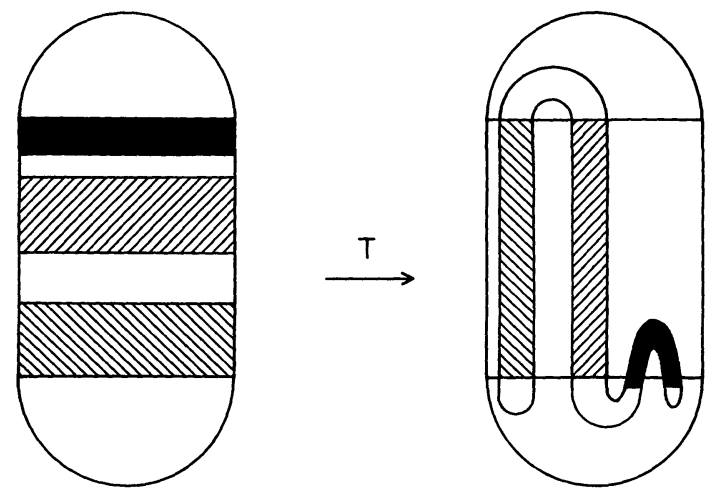

Yorke(1986) (see below). In the present work we would like to pursue this issue and provide a more complete, measurable characterization of the Newhouse set.

We start by observing that the essence of the Newhouse phenomenon is the creation of a hyperbolic chaotic state (by the principal homoclinicity to a saddle periodic orbit), then the realization of secondary homoclinicity to this latter state. Presumably this occurs as well in the homoclinic system to a Shil'nikov saddlefocus equilibrium point. Besides, the basic hyperbolic chaotic state may also be formed merely via a logistic map-like bifurcation scenario (Gaspard and Wang, 1987). Hence, it is of interest to consider a simple albeit mostly general situation, namely the homoclinic tangency to a given chaotic state (Wang, 1987; TedeschiniLalli and Yorke, 1986). In the present work, we propose to study a class of such systems, i.e. the homoclinicity to a standard Smale horseshoe. Figure 1 displays an example of the kind of planar diffeomorphisms we intend to investigate. Models of related type have been discussed in Gambaudo and Tresser (1983), in which an example of infinitely many sinks is explicitly given. Our system, which will be presented in Sect. 2, is constructed by extending the approach of Gavrilov and Shil'nikov which was initially developed in the case of homoclinic tangency to a periodic point of saddle type $(1972,1973)$. It consists of two locally defined mappings: one of them, $T_{0}(2.2)$, is defined near the basic invariant state $\Lambda$, and is assumed to be linearized; whereas the other, $T_{1}(2.5)$, brings outgoing orbits back to the neighborhood of $\Lambda$. Following Tedeschini-Lalli and Yorke (1986), we shall call the stable fixed points of $T_{1} \circ T_{0}^{n}$ simple sinks. The parameter set with infinitely many coexisting simple sinks is called the simple Newhouse set, which we denote by $J_{\infty}$ according to Robinson (1983).

The work of Tedeschini-Lalli and Yorke (1986) shows that $J_{\infty}$ is of zero Lebesgue measure. Our main purpose is to show that $J_{\infty}$ has a positive Hausdorff dimension $D_{H}\left(J_{\infty}\right)$. More precisely, we shall prove that

$$
\frac{\log 2}{2 \log \lambda_{u}}\left(1+\frac{\log \lambda_{u}}{\log \lambda_{s}^{-1}}\right)^{-1} \leqq D_{H}\left(J_{\infty}\right) \leqq \frac{\log 2}{2 \log \lambda_{u}} .
$$

with $2<\lambda_{u}<\lambda_{s}^{-1}$.

In Eq. (1.1) $\lambda_{u}$ (respectively $\lambda_{s}$ ) is the expanding (respectively contracting) rate of the basic state $\Lambda . \log 2$ is the topological entropy of the basic chaotic state $\Lambda$, and 
the factor $1 / 2$ comes from the assumption that the homoclinic tangency is quadratic.

This result is derived by relatively direct calculations which will be presented below step by step in a few short sections. A notable exception is Lemma 2 which is a crucial, and not so evident assertion; its the proof is given in the Appendix.

Let us comment on the meaning of formula (1.1). On one hand, the Tedeschini-Lalli and Yorke result is recovered directly from the formula (1.1), since the latter gives to $D_{\infty}\left(J_{\infty}\right)$ an upper bound smaller than the unit, and the fact that the Hausdorff dimension is less than one implies the nullness of the set. On the other hand, the lower bound is strictly positive. This conclusion will remain valid even if, when nonsimple Newhouse sinks are taken into account, the total Newhouse parameter set would turn out to be of positive Lebesgue measure.

We may thus conclude that the simple Newhouse set $J_{\infty}$ is a "fractal" in Mandelbrot's sense that its Hausdorff dimension is noninteger, and larger than its topological dimension. We remind the reader that $J_{\infty}$ is otherwise unlike the Cantor ternary set (a standard example of fractal) which is totally disconnected, nowhere dense, and which belongs to Baire's first category.

\section{The Homoclinic System to a Smale Horseshoe}

Consider a planar diffeomorphism $T(\cdot, \mu): \mathfrak{R}^{2} \mapsto \mathfrak{R}^{2}$, depending on a parameter $\mu$. We assume that $T(\cdot, \mu)$ realizes a standard Smale horseshoe (Smale, 1967), and is linearized on two horizontal strips (cf. Fig. 2a)

$$
H_{0}=I \times\left[0, \lambda_{u}^{-1}+\delta\right], \quad H_{1}=I \times\left[1-\lambda_{u}^{-1}-\delta, 1\right] .
$$

where $I \supset[0,1]$ and $\delta$ may be chosen arbitrarily small, and

$$
D(T)(x, y)=(-1)^{\omega}\left(\begin{array}{cc}
\lambda_{s} & 0 \\
0 & \lambda_{u}
\end{array}\right) \text { if } \quad(x, y) \in H_{\omega}, \omega=0,1,
$$

where $0<\lambda_{s}<\lambda_{u}^{-1}<1 / 2$.

Fig. 2 a

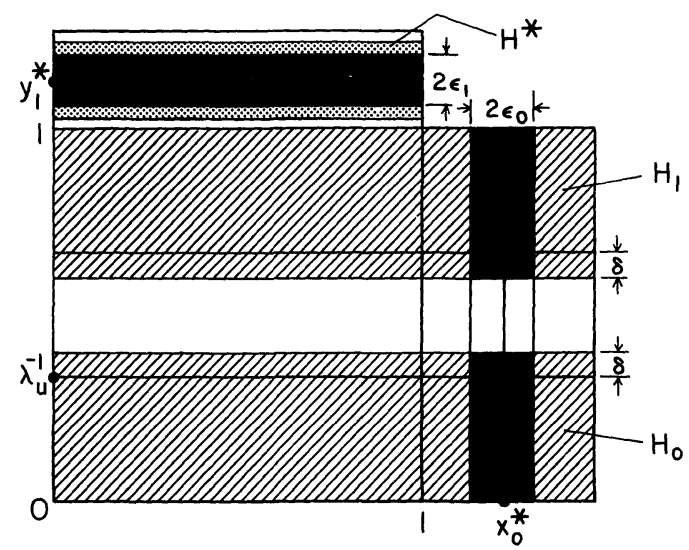


We denote by $T_{0}$ the mapping $T$ restricted on $H_{0} \cup H_{1}$. $T_{0}$ takes the form

$$
\begin{gathered}
\left\{\begin{array}{l}
x_{1}=\lambda_{s} x_{0} \\
y_{1}=\lambda_{u} y_{0}
\end{array} \text { if } \quad\left(x_{0}, y_{0}\right) \in H_{0}\right. \\
\left\{\begin{array}{l}
x_{1}=1-\lambda_{s} x_{0} \\
y_{1}=\lambda_{u}\left(1-y_{0}\right)
\end{array} \text { if } \quad\left(x_{0}, y_{0}\right) \in H_{1}\right.
\end{gathered}
$$

with $0<\lambda_{s}<\lambda_{u}^{-1}<1 / 2$.

We note that since $T_{0}$ is hyperbolic on $H_{0} \cup H_{1}$, it is insensitive to the parameter variation, thus its dependence on $\mu$ is disregarded in (2.2).

The nonwanding set $\Lambda$ of $T_{0}$ is a $2 D$ Cantor set, each point of which may be represented by a bi-infinite sequence $R=\left(\ldots \omega_{-2} \omega_{-1} \omega_{0} \omega_{1} \omega_{2} \ldots\right)$ of symbols $\omega_{i}=0$ or 1 (Smale, 1967). The local stable manifold $W_{\varepsilon}^{s}$ of $\Lambda$ consists of the product of a vertical Cantor set with a horizontal segment. The Cantor set has a Hausdorff dimension $D_{H}^{s}$ and a thickness $\tau^{s}$ given by

$$
D_{H}^{s}=\frac{\log 2}{\log \lambda_{u}} ; \quad \tau^{s}=\frac{\lambda_{u}^{-1}}{1-2 \lambda_{u}^{-1}} .
$$

The notion of the thickness of a linear Cantor set was introduced by Newhouse and played an important role in his work. For a general definition see Newhouse (1979), or Guckenheimer and Holmes (1983). The thickness of a standard ternary Cantor set is just the ratio of the length of one of the two remaining components to that of the middle gap, at any step of construction.

Similarly, for the local unstable manifold $W_{\varepsilon}^{u}$ which consists of the product of a horizontal Cantor set with a vertical segment, we have

$$
D_{H}^{u}=\frac{\log 2}{\log \lambda_{s}^{-1}} ; \quad \tau^{u}=\frac{\lambda_{s}}{1-2 \lambda_{s}} .
$$

The hyperbolic set $A$ of $T$ is not attracting. Almost every point in the unit square eventually enters into the horizontal strip $[0,1] \times\left[\lambda_{u}^{-1}, 1-\lambda_{u}^{-1}\right]$ after a finite number of iterations, before escaping from the unit square at the next step. We assume that there is an integer $k$, such that $T^{k}$ maps the rectangle $H^{*} C I$ $\times\left[1,1+\delta \lambda_{u}\right]$ back to the vicinity of $\Lambda, H_{0} \cup H_{1}$. (Please see Fig. 1 for an example with $k=1$, in which the black rectangle plays the role analogue to $H^{*}$.) Specifically, we suppose that in a small neighborhood of a horizontal curve $y=v(x, \mu)$ inside $H^{*}$, $T^{k}$ may be written as

$$
\begin{aligned}
& x_{0}=x_{0}^{*}+F\left(x_{1}, y_{1}-v\left(x_{1}, \mu\right), \mu\right), \\
& y_{0}=G\left(x_{1}, y_{1}-v\left(x_{1}, \mu\right), \mu\right)
\end{aligned}
$$

with $F(0,0,0)=0 ; \quad-b=\partial F / \partial y(0,0,0) \neq 0 ; \quad G(0,0,0)=0 ; \quad c=\partial G / \partial x(0,0,0) \neq 0$; $\partial G / \partial y(0,0,0)=0$; and $d=\partial^{2} G / \partial y^{2}(0,0,0) \neq 0$. And $x_{0}^{*} \in I$ may be chosen arbitrarily near to, albeit larger than the unit.

At the lowest approximation, $v\left(x_{1}, \mu\right) \equiv y_{1}^{*}=$ constant; and the mapping $T^{k}$ restricted on a small rectangle with $\left|y_{1}-y_{1}^{*}\right| \leqq \varepsilon_{1}$ is denoted as $T_{1}$ (Fig. 2b). The simplest form that $T_{1}$ can take while fulfilling (2.4) is

$$
x_{0}=x_{0}^{*}-b\left(y_{1}-y_{1}^{*}\right), \quad y_{0}=\mu+c x_{1}+\frac{d}{2}\left(y_{1}-y_{1}^{*}\right)^{2} .
$$


Fig. 2b

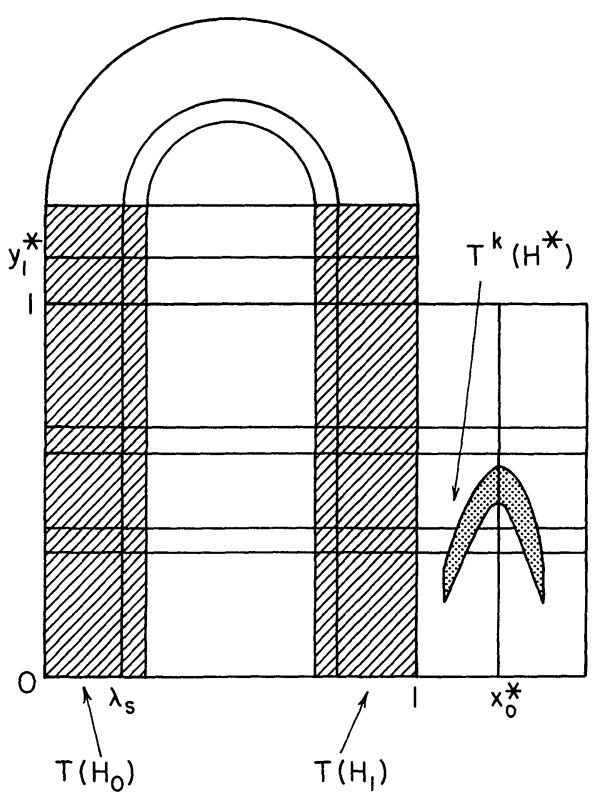

with $1<y_{1}^{*}<1+\delta \lambda_{u}$, Jacobian $\left(T_{1}\right)=c b>0$. One sees from (2.5) that any vertical straight line in $H^{*}$ (with fixed $x_{1}$ ) will be mapped by $T_{1}$ into a parabola with the extremum located at $x_{0}=x_{0}^{*}$ (Fig. 2b). In order to satisfy $\left|x_{0}-x_{0}^{*}\right| \leqq \varepsilon_{0}$ for all $y_{1}:\left|y_{1}-y_{1}^{*}\right| \leqq \varepsilon_{1}$, it is sufficient to choose $\varepsilon_{1}=\varepsilon_{0} /|b|$.

We have therefore constructed two mappings: the $T_{0}(2.2)$ which has $H_{0} \cup H_{1}$ as its definition domain; and $T_{1}(2.5)$ which has $H^{*}$ as its definition domain. Let

$$
\Pi_{0}=\left\{\left|x_{0}-x_{0}^{*}\right| \leqq \varepsilon_{0}, y_{0} \in\left[0, \lambda_{u}^{-1}+\delta\right] \cup\left[1-\lambda_{u}^{-1}-\delta, 1\right]\right\} \subset H_{0} \cup H_{1}
$$

and

$$
\Pi_{1}=\left\{x_{1} \in[0,1],\left|y_{1}-y_{1}^{*}\right| \leqq \varepsilon_{1}\right\} \subset H^{*} .
$$

Clearly, $T_{1}: \Pi_{1} \rightarrow \Pi_{0}$ is well defined provided that $\mu$ is confined in a suitable interval. Such constraint on $\mu$ will be specified in the subsequent section. In order to complete the construction of our system, it remains to show that $T_{0}^{l}: \Pi_{0} \rightarrow \Pi_{1}$ is equally well defined for any $l$. Let $S_{l}=\omega_{0} \omega_{1} \ldots \omega_{l-1}$ be a finite string of zeros and ones. $T_{0}^{l}$ denotes the $l$ th-iteration of $T_{0}$ according to $S_{l}, T_{0}^{j}(p) \in H_{\omega_{j}}, 0 \leqq j \leqq l-1$. Then, we have [cf. (2.2)]

$$
\left(x_{1}, y_{1}\right)=T_{0}^{l}\left(x_{0}, y_{0}\right)=\left(E_{l}+F_{l} \cdot x_{0}, C_{l}+D_{l} \cdot y_{0}\right),
$$

with

$$
\begin{aligned}
& E_{l}=\omega_{l-1}+(-1)^{\omega_{l-1}} \omega_{l-2} \lambda_{s}+\ldots+(-1)^{\omega_{l-1}+\ldots+\omega_{1}} \omega_{0} \lambda_{s}^{l-1}, \\
& F_{l}=\lambda_{s}^{l}(-1)^{\omega_{0}+\omega_{1}+\ldots+\omega_{l-1}} \\
& C_{l}=\omega_{l-1} \lambda_{u}+(-1)^{\omega_{l-1}} \omega_{l-2} \lambda_{u}^{2}+\ldots+(-1)^{\omega_{l-1}+\ldots+\omega_{1}} \omega_{0} \lambda_{u}^{l}, \\
& D_{l}=\lambda_{u}^{l}(-1)^{\omega_{0}+\omega_{1}+\ldots+\omega_{l-1}} .
\end{aligned}
$$


Fig. 3

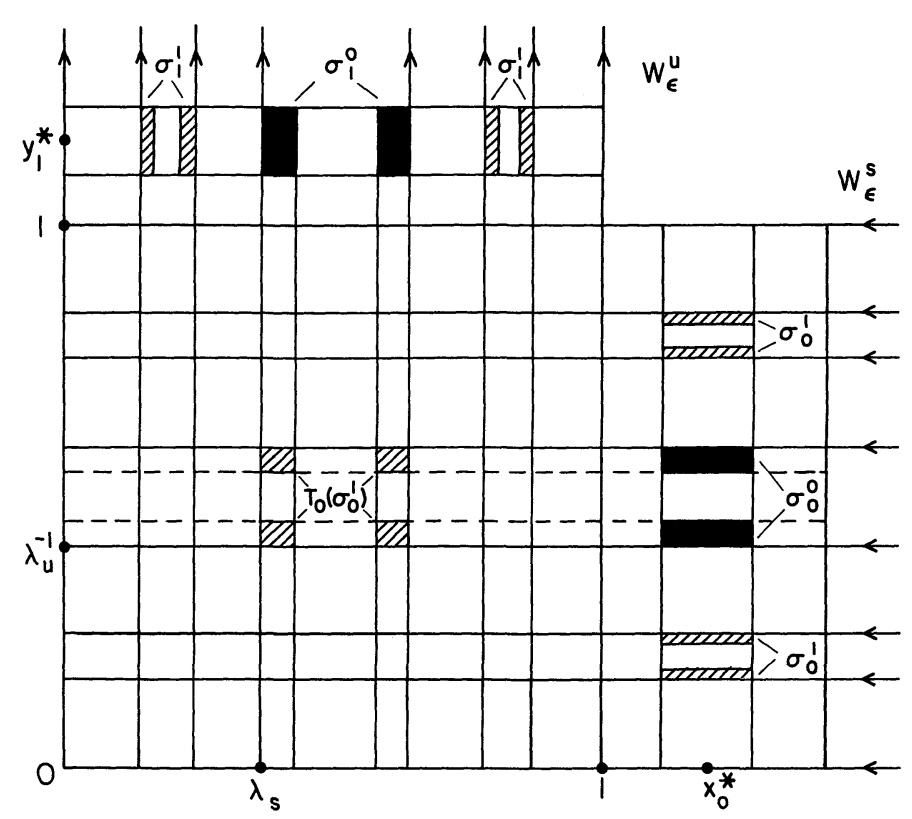

Equation (2.6) may be rewritten as

$$
\begin{aligned}
& x_{1}=\zeta\left(x_{0}, y_{1} ; S_{l}\right) \equiv E_{l}+F_{l} \cdot x_{0}, \\
& y_{0}=\eta\left(x_{0}, y_{1} ; S_{l}\right) \equiv \frac{y_{1}-C_{l}}{D_{l}} .
\end{aligned}
$$

The definition domain $\sigma_{0}^{l}$ of $T_{0}^{l}: \Pi_{0} \rightarrow \Pi_{1}$ is given as the image of the rectangle

$$
\left\{\left|x_{0}-x_{0}^{*}\right| \leqq \varepsilon_{0},\left|y_{1}-y_{1}^{*}\right| \leqq \varepsilon_{1}\right\}
$$

under the action of the linear transformation defined by

$$
x_{0}=x_{0}, \quad y_{0}=\eta\left(x_{0}, y_{1} ; S_{l}\right) \text {. }
$$

It can be easily seen that each $\sigma_{0}^{l}$ is a small horizontal box in $\Pi_{0}$, and the ensemble $\left\{\sigma_{0}^{l}\right\}$ is composed of a countably infinite number of disjoint horizontal boxes included in $\Pi_{0}$. In fact, if $S_{l} \neq S_{k}^{\prime}$, then $T_{0}^{i}\left(\sigma_{0}^{l}\right) \cap T_{0}^{j}\left(\sigma_{0}^{k}\right)=\emptyset, i=0,1, \ldots, l-1$, and $j=0,1, \ldots, k-1$. See Fig. 3 for an illustration with $\sigma^{l}, l=0,1$ and their images by $T_{0}^{k}$, $k=0,1, \ldots, l-1$. One notices that they are arbitrarily near to the basic invariant state $A$.

The image of $\sigma_{0}^{l}$ by $T_{0}^{l}, \sigma_{1}^{l}=T_{0}^{l} \sigma_{0}^{l}$, is also that of the rectangle (2.9) under the action of the linear transformation defined by

$$
x_{1}=\zeta\left(x_{0}, y_{1} ; S_{l}\right), \quad y_{1}=y_{1} .
$$

One can similarly check that the ensemble $\left\{\sigma_{1}^{l}\right\}$ is composed of a countably infinite number of disjoint vertical boxes included in $\Pi_{1}$. Therefore, $T_{0}^{l}: \Pi_{0} \rightarrow \Pi_{1}$ is indeed well defined for all $l$. 


\section{The Persistence of Homoclinic Tangencies}

In this section we specify the conditions under which homoclinic tangencies to the invariant state $\Lambda$ of the system (2.2), (2.5) persist in a whole parameter interval of $\mu$. The possibility of such robustness will be of central importance.

Let $\Gamma_{s}=\left(\left\{x_{0}^{*}\right\} \times[0,1]\right) \cap W_{\varepsilon}^{s}$ and $\Gamma_{u}=\left([0,1] \times\left\{y_{1}^{*}\right\}\right) \cap W_{\varepsilon}^{u}$. It is obvious that $T_{1}$ maps the horizontal Cantor set $\Gamma_{u}$ (with $\left.y_{1}=y_{1}^{*}\right)$ into a vertical Cantor set $T_{1}\left(\Gamma_{u}\right)$ of the parabolic extrema (with $x_{0}=x_{0}^{*}$ ). Since this is a simple affine transformation [cf. (2.5)], $x_{0}=x_{0}^{*}, y_{0}=\mu+c x_{1}, T_{1}\left(\Gamma_{u}\right)$ has the same Hausdorff dimension and thickness as $\Gamma_{u}$. Both $\Gamma_{s}$ and $T_{1}\left(\Gamma_{u}\right)$ are vertical linear Cantor sets located at $x_{0}=x_{0}^{*}$. As $\mu$ varies, the local stable manifold $W_{\varepsilon}^{s}$ and the global unstable manifold $T_{1}\left(W_{\varepsilon}^{u}\right)$ can intersect with each other, to creat homoclinic orbits. A homoclinic tangency is realized each time $T_{1}\left(\Gamma_{u}\right) \cap \Gamma_{s} \neq \emptyset$. We have the following

Proposition 1. If $\tau\left(\Gamma_{s}\right) \cdot \tau\left(\Gamma_{u}\right)>1$, i.e.

$$
\left(\lambda_{s}-1 / 2\right)\left(\lambda_{u}-3 / 2\right)+1 / 4>0,
$$

then, $T_{1}\left(\Gamma_{u}\right) \cap \Gamma_{s} \neq \emptyset$ for a whole interval of the values of $\mu$.

Proposition 1 is an immediate consequence of the Newhouse lemma

Lemma 1 (Newhouse, 1979). Let $F$ and $G$ be two Cantor sets in $\Re$ with $F$ in no G-gap closure and $G$ in no F-gap closure. If $\tau(F) \cdot \tau(G)>1$, then $F \cap G \neq \emptyset$.

In order to ensure the condition of the lemma, that $\Gamma_{s}$ is in no $T_{1}\left(\Gamma_{u}\right)$-gap closure and $T_{1}\left(\Gamma_{u}\right)$ is in no $\Gamma_{s}$-gap closure, a parameter domain of $\mu$ should be chosen appropriately. Since $T_{1}\left(\Gamma_{u}\right)$ is contained in the interval between $\mu$ and $\mu+c$, we see that if

$$
|c|>1-2 \lambda_{u}^{-1}
$$

$T_{1}\left(\Gamma_{u}\right)$ well not be found inside any gap of $\Gamma_{s}$. Then, the following conditions on $\mu$ :

$$
\mu \in[-c, 1] \text { if } c>0 ; \text { or } \mu \in[0,1-c] \text { if } c<0
$$

guarantee the condition of the Lemma 1 to be fulfilled. For mere convenience we shall suppose (3.2) to be true for $c$, an alternation of which should not affect the main conclusions of our analyses; and henceforth we let $c>0 . \lambda_{s}, \lambda_{u}$ will be assumed to satisfy both (2.2) and (3.1) (Fig. 4). Notice that the product $\tau\left(\Gamma_{s}\right) \cdot \tau\left(\Gamma_{u}\right)$ can be

Fig. 4

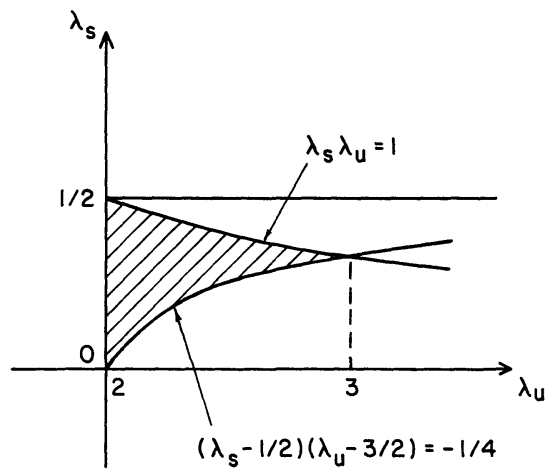


rendered as large as we please, by approaching $\lambda_{u} \rightarrow 2$; while still maintaining (2.2), so that the basic set $\Lambda$ remains hyperbolic.

\section{Coexistence of Infinitely Many Simple Sinks}

The simple periodic solutions are fixed points of $T_{1} \circ T_{0}^{l}$, i.e. [cf. (2.5)-(2.6)]

$$
\begin{aligned}
& x_{0}=x_{0}^{*}-b\left(C_{l}+D_{l} y_{0}-y_{1}^{*}\right), \\
& y_{0}=\mu+c\left(E_{l}+F_{l} x_{0}\right)+\frac{d}{2}\left(C_{l}+D_{l} y_{0}-y_{1}^{*}\right)^{2} .
\end{aligned}
$$

Substituting $x_{0}$ in (4.1b) by (4.1a), we obtain

$$
\mu=y_{0}-c\left[E_{l}+F_{l}\left(x_{0}^{*}-b\left(C_{l}+D_{l} y_{0}-y_{1}^{*}\right)\right)\right]-\frac{d}{2}\left(C_{l}+D_{l} y_{1}-y_{1}^{*}\right)^{2} .
$$

A saddle-node bifurcation occurs when $\partial \mu / \partial y_{0}=0$. Two solutions bifurcate in this way, one is stable, the other is unstable. They can be given symbolic names $\left(S_{l} \pm\right)$, where \pm specify $y_{1}<$ or $>y_{1}^{*}$. We are interested in the stable solution, which we denote by $\left(S_{l} H\right)$, with $H=+$ or - . Let $\lambda_{1}$ and $\lambda_{2}$ be the two eigenvalues of this solution, then, it follows from straightforward computations that

$$
\begin{gathered}
\text { at } \mu_{l}^{t}=\frac{y_{1}^{*}-C_{l}}{D_{l}}-c\left(E_{l}+F_{l} x_{0}^{*}\right)+\Delta_{l} / 2, \\
\lambda_{1}=+1, \quad 0<\lambda_{2}<1, \\
\text { at } \quad \mu_{l}^{*}=\frac{y_{1}^{*}-C_{l}}{D_{l}}-c\left(E_{l}+F_{l} x_{0}^{*}\right), \\
\lambda_{1,2}= \pm i \Omega, \quad 0<\Omega=\sqrt{c b\left(\lambda_{s} \lambda_{u}\right)^{l}}<1, \\
\text { at } \quad \mu_{l}^{h}=\frac{y_{1}^{*}-C_{l}}{D_{l}}-c\left(E_{l}+F_{l} x_{0}^{*}\right)-3 \Delta_{l} / 2, \\
\lambda_{1}=-1, \quad-1<\lambda_{2}<0
\end{gathered}
$$

with $\Delta_{l}=\frac{\left(c b D_{l} F_{l}+1\right)^{2}}{d D_{l}^{2}}$.

That is, $\left(S_{l} H\right)$ becomes unstable at $\mu=\mu_{l}^{h}$, via a period doubling bifurcation. Its range of its stability, or the window width in the $\mu$-space, is

$$
\left|\mu_{l}^{t}-\mu_{l}^{h}\right|=2\left|\Delta_{l}\right| \sim_{l \rightarrow \infty} \lambda_{u}^{-2 l} .
$$

We have therefore obtained the explicit expressions for all the simple periodic windows in the $\mu$-space. With this knowledge we can now show that infinitely many of them may coexist in the system (2.2), (2.5). Firstly, we have the following

Proposition 2. Under the conditions (2.2) and (3.1) on $\lambda_{s}$ and $\lambda_{u}$, and (3.2) with $c>0$, the set $\left\{\mu_{l}^{t}\right\}$ is dense in $[-c, 1]$.

Proof. By virtue of the Proposition 1, which is valid if (2.2) and (3.1)-(3.2) are fulfilled, $\forall \mu \in[-c, 1], \exists\left(x_{0}^{*}, y^{*}\right) \in \Gamma_{s}$, and $\left(x^{*}, y_{1}^{*}\right) \in \Gamma_{u}$, such that

$$
T_{1}\left(x^{*}, y_{1}^{*}\right)=\left(x_{0}^{*}, y^{*}\right) \text { or } y^{*}=\mu+c x^{*},
$$


since $\left(x_{0}^{*}, y^{*}\right) \in \Gamma_{s}$, there are horizontal gaps arbitrarily near $\left(x_{0}^{*}, y^{*}\right) . \forall \varepsilon>0$, there exists a gap to which $\left(x_{0}, y_{l}\right)$ belongs, $y_{l}=\left(y_{1}^{*}-C_{l}\right) / D_{l}$ and $l$ larger enough, such that

$$
\left|y_{l}-y^{*}\right|<\frac{\varepsilon}{2} \quad \text { and } \quad c\left(1+x_{0}^{*}\right) \lambda_{s}^{l}<\frac{\varepsilon}{2} .
$$

Let $x^{*}=E_{l}+F_{l} \tilde{x}^{*}, \tilde{x}^{*} \in[0,1]$. We have

$$
\begin{aligned}
\left|\mu-\mu_{l}^{*}\right| & =\left|y^{*}-c x^{*}-\frac{y_{1}^{*}-C_{l}}{D_{l}}+c\left(E_{l}+F_{l} x_{0}^{*}\right)\right| \\
& \leqq\left|y^{*}-\frac{y_{1}^{*}-C_{l}}{D_{l}}\right|+c\left|F_{l}\right|\left|\tilde{x}^{*}-x_{0}^{*}\right| \\
& \leqq \frac{\varepsilon}{2}+\frac{\varepsilon}{2}=\varepsilon .
\end{aligned}
$$

Hence, $\left\{\mu_{l}^{*}\right\}$ is dense in $[-c, 1]$, so is $\left\{\mu_{l}^{t}\right\}$.

Remark. This proposition may be understood easily in terms of the two basic ingredients of the mechanism giving rise to the Newhouse phenomenon. According to the Proposition $1, \Gamma_{s} \cap T_{1}\left(\Gamma_{u}\right) \neq \emptyset$, thus there is a homoclinic tangency, for every $\mu \in[-c, 1]$. The point $\left(x^{*}, y^{*}\right)$ satisfying $(4.5)$, for which the tangency is realized, changes as $\mu$ is varied. Now we know that the periodic orbits are dense in the invariant basic set $\Lambda$, so are their local stable (respectively unstable) manifolds in $W_{\varepsilon}^{s}$ (respectively $W_{\varepsilon}^{u}$ ). Consequently, when $\mu$ is varied continuously, for a dense set of $\mu$ values in $[-c, 1]$, the corresponding $\left(x^{*}, y^{*}\right)$ belongs to a periodic orbit $S_{l}$ of $\Lambda$ and the tangency is just homoclinic tangency to this periodic orbit of saddle type. Here enters the second ingredient: arbitrarily near such a tangency there exist saddle-node bifurcation cascades; each resulting sink with its stability range given by (4.4) (see e.g. Gavrilov and Shl'nikov 1972, 1973). It thus follows that saddle-node bifurcation points are dense in $[-c, 1]$, which is the assertion of the Proposition 2.

Corollary. There is a residual set $J_{\infty} \subset[-c, 1]$, for which the system (2.2), (2.5) has simultanously an infinite number of simple sinks.

Proof. The argument is adopted from Robinson (1983). Since $\left\{\mu_{l}^{t}\right\}$ is dense, the set $J_{1}$ of $\mu$ values with one sink is open and dense. And since $\left\{\mu_{l}^{t}\right\}$ is dense in $J_{1}$, the set $J_{2}$ with two coexisting sinks is open dense, $J_{1} \supset J_{2}$. Repeating the argument ad infinitum, we get a sequence of dense open sets, $J_{1} \supset J_{2} \supset \ldots \supset J_{\infty}$, the limit of which is a residual set, $J_{\infty}$, with infinitely many coexisting simple sinks.

\section{The Hausdorff Dimension of $J_{\infty}$}

The preceding computations enable us to estimate now the Hausdorff dimension of the simple Newhouse set $J_{\infty}$. The key assertion is the following

Lemma 2. For l large enough, there are $\sim 2^{\text {hl }}$, where

$$
h=\left(1+\log \lambda_{u} / \log \lambda_{s}^{-1}\right)^{-1}
$$



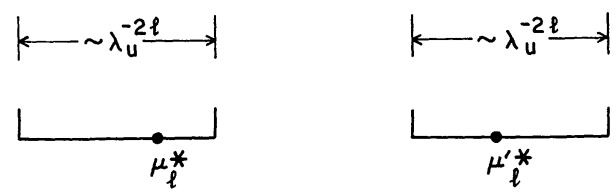

Fig. 5

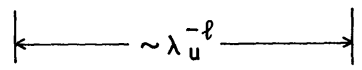

among the $\sim 2^{l}$ windows, all with the same period and same window width, which do not overlap with each other in the $\mu$-space.

The proof of this lemma, which is somewhat long and will be given in the appendix, is based on the fact that, for any pair of such specially selected periodic windows, the distance between $\mu_{l}^{*}$ and $\mu_{l}^{\prime *}$ behaves as $\sim \lambda_{u}^{-l}$ up to a certain polynomial correction in $l$; while the width of the windows is of order $\lambda_{u}^{-2 l}$ (Fig. 5).

By definition of $J_{\infty}$, we see that $\mu \in J_{\infty}$ iff $\forall l \geqq 1, \exists l^{\prime} \geqq l$, and $\mu_{l}^{\prime *}$ such that

$$
\mu \in\left(\mu_{l}^{\prime *}+\Delta_{l}^{\prime} / 2, \mu_{l}^{\prime *}-3 \Delta_{l}^{\prime} / 2\right) \text {. }
$$

(For definiteness, and in accordance with Fig. 2, we choose $d<0$, so that $\Delta_{l}^{\prime}<0$.) Let

$$
G_{n}=\bigcup_{l \geqq n} \bigcup_{S_{l}}\left(\mu_{l}^{*}+\Delta_{l} / 2, \mu_{l}^{*}-3 \Delta_{l} / 2\right)
$$

then

$$
J_{\infty}=\bigcap_{n} G_{n} .
$$

Being a residual set, $J_{\infty}$ is uncountable and dense in $[-c, 1]$. We shall now show that it has a positive Hausdorff dimension. Let us recall

Definition 1 (Billingsley, 1978). Consider an arbitrary bounded set $A$ in $\mathfrak{R}$. For $s$, $\varepsilon>0$, let

$$
m_{s}^{\varepsilon}(A)=\inf \sum_{j}|I|_{j}^{s}, \quad \bigcup_{j} I_{j} \supset A, \quad\left|I_{j}\right|<\varepsilon,
$$

where the infimum is taken over all countable coverings $\left\{I_{j}\right\}$ of $A$,

$$
m_{s}(A)=\lim _{\varepsilon \rightarrow 0} m_{s}^{\varepsilon}(A) \text {. }
$$

Then, there exists a unique $D_{H}(A)$, which is called the Hausdorff dimension of the set $A$, such that

$$
\begin{aligned}
& \text { if } s<D_{H}(A), \quad m_{s}(A)=\infty \text {, } \\
& \text { if } \quad s>D_{H}(A), \quad m_{s}(A)=0 \text {. }
\end{aligned}
$$

For the simple Newhouse set $J_{\infty}$, we have the following

\section{Proposition 3.}

$$
\frac{\log 2}{2 \log \lambda_{u}}\left(1+\frac{\log \lambda_{u}}{\log \lambda_{s}^{-1}}\right)^{-1} \leqq D_{H}\left(J_{\infty}\right) \leqq \frac{\log 2}{2 \log \lambda_{u}}
$$


Proof. First, we observe that

$$
\begin{aligned}
m_{s}\left(G_{n}\right) & \leqq \sum_{l=n}^{\infty} 2^{l}\left(2 \Delta_{l}\right)^{s} \leqq\left[\frac{2(1+c b)}{d}\right]^{s} \sum_{l=n}^{\infty}\left(2 / \lambda_{u}^{2 s}\right)^{l} \\
& =\left[\frac{2(1+c b)}{d}\right]^{s}\left[\frac{2 / \lambda_{u}^{2 s}}{1-2 / \lambda_{u}^{2 s}}\right]^{n} \rightarrow 0 \text { if } 2 / \lambda_{u}^{2 s}<1
\end{aligned}
$$

Therefore, $m_{s}\left(J_{\infty}\right)=0$, if $s>\frac{\log 2}{2 \log \lambda_{u}}$.

On the other hand, Lemma 2 implies that every $G_{n}$ will contain $\sim 2^{h l}$ [with $h$ given in (5.1)] disjoint opens for sufficiently large $l$, each with a width $2\left|\Delta_{l}\right|, l \geqq n$. Consequently,

$$
m_{s}\left(G_{n}\right) \geqq 2^{l h}\left(2 \Delta_{l}\right)^{s} \geqq(2 / d)^{s}\left(2^{h} / \lambda_{u}^{2 s}\right)^{n} \rightarrow \infty \quad \text { if } \quad 2^{h} / \lambda_{u}^{2 s}>1
$$

Therefore, $m_{s}\left(J_{\infty}\right)=\infty$, if $s<\frac{h \log 2}{2 \log \lambda_{u}}$.

And the proposition follows from Definition 1, with $h$ given in (5.1).

\section{Concluding Remarks}

In this paper we have discussed the question raised in the introduction concerning the measurable properties of the parameter set corresponding to the coexistence of infinitely many sinks in the phase space of a dynamical system. Our analysis in the previous sections was limited to the simple Newhouse sinks as the stable fixed points of $T_{1} \circ T_{0}^{l}$. In Wang (1987) nonsimple Newhouse sinks have also been investigated. It seems worthwhile to mention some conclusion drawn from there.

In general we shall call a Newhouse sink a stable fixed point of

$$
T_{1} \circ T_{0}^{l_{k}} \circ T_{1} \circ T_{0}^{l_{k-1}} \circ \ldots \circ T_{1} \circ T_{0}^{l_{1}} .
$$

The complete Newhouse set denoted by $J_{N}$, is the set of parameter values for which the system has infinitely many coexisting Nowhouse sinks. Let us divide the total Newhouse set $J_{N}$ into two disjoint subsets: $J_{N}=J_{A} \cup J_{B} . J_{B}$ is defined as follows: let it be given any large integer $M$. For $\mu \in J_{N}$, let the associated, countable set of coexisting sinks be

$$
\Omega=\left\{\left(S_{l_{1}} \varepsilon_{1} S_{l_{2}} \varepsilon_{2} \ldots \varepsilon_{l_{k_{j}-1}} S_{l_{k_{j}}} H_{j}\right)\right\}_{j=1}^{\infty}, \quad S_{l_{r}}=\left(\omega_{0} \ldots \omega_{l_{i}}\right), \quad \varepsilon_{i}= \pm 1
$$

Then, $\mu \in J_{B}$ if only a finite number of elements in the sequence $\Omega(6.1)$, contain certain $S_{l_{i}}$ longer than $M, l_{i}>M$; and all the infinitely many others in the sequence involve only the $S_{l_{l}}$ of length shorter than $M, l_{i}<M$. It then follows from the infinitude of the collection $\Omega$ that the sequence $\left\{k_{j}, j=1,2,3, \ldots\right\}$ must be unbounded. In other words, the largest period amongst these $\operatorname{sinks}$ in $\Omega$ tends to the infinity via $k \rightarrow \infty$ instead of $l \rightarrow \infty$, in contrast to the case of $J_{\infty}$. Obviously the larger is $M$, the larger is the set $J_{B}$. The complement of $J_{B}$ is denoted by $J_{A}$, $J_{A}=J_{N} \backslash J_{B}$. Evidently, we have $J_{\infty} \subset J_{A}$.

Nothing is known about the Newhouse subset $J_{B}$, not even whether it is a nonempty set. On the other hand, since $J_{\infty} \subset J_{A}$, it follows immediately from the 
previous sections that the Hausdorff dimension of $J_{A}$ is strictly positive. Indeed, $J_{A}$ enjoys quite similar properties as $J_{\infty}$. By extending the techniques developed in Tedeschini-Lalli and Yorke (1986), we can show that

Proposition 4 (Wang, 1987). There exists an $M$ such that the Lebesgue measure of $J_{A}$ is zero.

The proof will not be given here. Let us end by saying that in order to provide with a complete answer concerning the Lebesgue measure of the Newhouse set $J_{N}$, it remains to understand the other Newhouse subset $J_{B}$.

\section{Appendix: Proof of the Lemma 2}

We would like to show that as $l \rightarrow \infty$, there are about $2^{h l}$ simple windows, with $h=\left(1+\frac{\log \lambda_{u}}{\log \lambda_{s}^{-1}}\right)^{-1}$, out of the total $\sim 2^{l}$ simple windows of same period, which do not overlap amongst themselves. To this end, consider any pair of two windows, $\left(\mu_{l}^{*}+\Delta_{l} / 2, \mu_{l}^{*}-3 \Delta_{l} / 2\right)$ and $\left(\mu_{l}^{\prime *}+\Delta_{l}^{\prime} / 2, \mu_{l}^{\prime *}-3 \Delta_{l}^{\prime} / 2\right)$ [cf. (4.3)]. They are separated if

$$
\begin{aligned}
\left|\mu_{l}^{*}-\mu_{l}^{\prime *}\right| & =\left|\frac{y_{1}^{*}-C_{l}}{D_{l}}-\frac{y_{1}^{*}-C_{l}^{\prime}}{D_{l}^{\prime}}-c\left(E_{l}+F_{l} x_{0}^{*}\right)+c\left(E_{l}^{\prime}+F_{l}^{\prime} x_{0}^{*}\right)\right| \\
& \geqq\left|\frac{y_{1}^{*}-C_{l}}{D_{l}}-\frac{y_{1}^{*}-C_{l}^{\prime}}{D_{l}^{\prime}}-c\right| E_{l}-E_{l}^{\prime}||-c x_{0}^{*}\left|F_{l}-F_{l}^{\prime}\right| \\
& >2\left|\Delta_{l}\right| .
\end{aligned}
$$

Let us suppose that these two sinks are stable fixed points of $T_{1} \circ T_{0}^{l}$ according to $S_{l}=\omega_{0} \omega_{1} \ldots \omega_{l-1}$, and $S_{l}^{\prime}=\omega_{0}^{\prime} \omega_{1}^{\prime} \ldots \omega_{l-1}^{\prime}$, respectively. We assume

$$
\begin{aligned}
\omega_{k}=\omega_{k}^{\prime}, \quad k=0,1, \ldots, i-1 ; & k=l-1, l-2, \ldots, j+1 \\
& \text { and } \quad \omega_{i} \neq \omega_{i}^{\prime}, \quad \omega_{j} \neq \omega_{j}^{\prime}
\end{aligned}
$$

$(i \leqq j)$. Then, $S_{l}$ and $S_{l}^{\prime}$ are separated by a distance larger than a gap $S_{i}$ (Fig. 6a). Recall that $\left(x_{0}^{*}, \frac{y_{1}^{*}-C_{l}}{D_{l}}\right)\left[\right.$ respectively $\left.\left(x_{0}^{*}, \frac{y_{1}^{*}-C_{l}^{\prime}}{D_{l}^{\prime}}\right)\right]$ is a point inside the gap $S_{l}$ (respectively $S_{l}^{\prime}$ ), we have

$$
\lambda_{u}^{-i} \geqq\left|\frac{y_{1}^{*}-C_{l}}{D_{l}}-\frac{y_{1}^{*}-C_{1}^{\prime}}{D_{l}^{\prime}}\right| \geqq \lambda_{u}^{-i}\left(1-2 \lambda_{u}^{-1}\right) .
$$

Similarly, recalling that [cf. (2.7)]

$$
E_{l}=\omega_{l-1}+(-1)^{\omega_{l-1}} \omega_{l-2} \lambda_{s}+\ldots+(-1)^{\omega_{l-1}+\ldots+\omega_{1}} \omega_{0} \lambda_{s}^{l-1},
$$

we have (cf. Fig. 6b)

$$
\lambda_{s}^{l-j-1} \geqq\left|E_{l}-E_{l}^{\prime}\right| \geqq \lambda_{s}^{l-j-1}\left(1-2 \lambda_{s}\right) .
$$

Let us select two different cases:

$$
\begin{aligned}
& \text { case (A) } \lambda_{u}^{-i}\left(1-2 \lambda_{u}^{-1}\right)>c \lambda_{s}^{l-j-1}, \\
& \text { case (B) } c \lambda_{s}^{l-j-1}\left(1-2 \lambda_{s}\right)>\lambda_{u}^{-i} .
\end{aligned}
$$




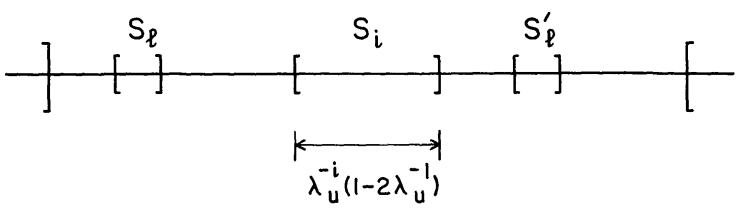

Fig. 6a


b

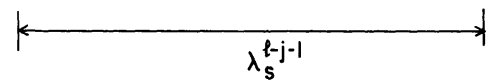

In case (A), it follows from (A.3) and (A.5) that $\left|\frac{y_{1}^{*}-C_{l}}{D_{l}}-\frac{y_{1}^{*}-C_{l}^{\prime}}{D_{l}^{\prime}}\right| \geqq c\left|E_{l}-E_{l}^{\prime}\right|$, hence a sufficient condition in order to satisfy (A.1) is

$$
\left|\mu_{l}^{*}-\mu_{l}^{\prime *}\right| \geqq c \lambda_{u}^{-i}\left(\tilde{c}-\lambda_{s}^{l-j-1} \lambda_{u}^{i}\right)-2 c x_{0}^{*} \lambda_{s}^{l}>2\left|\Delta_{l}\right|,
$$

where $\tilde{c}=\left(1-2 \lambda_{u}^{-1}\right) / c$.

Or in case (B), $\left|\frac{y_{1}^{*}-C_{l}}{D_{l}}-\frac{y_{1}^{*}-C_{l}^{\prime}}{D_{l}^{\prime}}\right| \leqq c\left|E_{l}-E_{l}^{\prime}\right|$, and a sufficient condition in order to satisfy (A.1) is

$$
\left|\mu_{l}^{*}-\mu_{l}^{*}\right| \geqq \lambda_{u}^{-i} / \tilde{c}^{\prime}\left(\lambda_{s}^{l-j-1} \lambda_{u}^{i}-\tilde{c}^{\prime}\right)-2 c x_{0}^{*} \lambda_{s}^{l}>2\left|\Delta_{l}\right|,
$$

where $\tilde{c}^{\prime}=\left(c\left(1-2 \lambda_{s}\right)\right)^{-1}$.

Now, we would like to show firstly that for large $l$, (A.6a) [respectively (A.6b)] implies (A.7) [respectively (A.8)], thus the corresponding pair of simple windows do not overlap (Proposition 5). Then, we shall estimate the number of windows in each of the two classes $A$ and $B$ specified respectively by (A.6a) and (A.6b) (Proposition 6).

Proposition 5. For $l$ large enough, (A.7) (respectively (A.8)) holds if (A.6a) (respectively (A.6b)) is fulfilled.

Proof. Consider firstly case A, for which we need to prove the truth of (A.7). We observe that for almost any arbitrarily chosen $c, \lambda_{s}$, and $\lambda_{u}$, the two real numbers $\log \lambda_{s} / \log \tilde{c}$ and $\log \lambda_{u} / \log \tilde{c}$, will satisfy the Diophantine condition (Chenciner and Iooss 1979): $\forall \varepsilon>0, \exists A^{\prime}>0$ such that

$$
\left|m \frac{\log \lambda_{s}}{\log \tilde{c}}+n \frac{\log \lambda_{u}}{\log \tilde{c}}-1\right|>\frac{A^{\prime}}{l^{2+\varepsilon}}, \quad \forall m, n \leqq l .
$$

Then, if $m \log \lambda_{s}+n \log \lambda_{u}-\log \tilde{c}<0$,

$$
\lambda_{s}^{m} \cdot \lambda_{u}^{n} / \tilde{c}<\exp \left(-A / l^{2+\varepsilon}\right)
$$


where $A=A^{\prime}|\log \tilde{c}|$. Consequently,

$$
\tilde{c}-\lambda_{s}^{m} \cdot \lambda_{u}^{n}>\tilde{c}\left(1-\exp \left(-A / l^{2+\varepsilon}\right)\right) \sim_{l \rightarrow \infty} \tilde{c} A / l^{2+\varepsilon} .
$$

On the other hand, in case (B), $m \log \lambda_{s}+n \log \lambda_{u}-\log \tilde{c}^{\prime}>0$,

$$
\left(\lambda_{s}^{m} \cdot \lambda_{u}^{n}\right) / \tilde{c}>\exp \left(A / l^{2+\varepsilon}\right)
$$

with $A=A^{\prime}\left|\log \tilde{c}^{\prime}\right|$ and,

$$
\lambda_{s}^{m} \lambda_{u}^{n}-\tilde{c}^{\prime}>\tilde{c}^{\prime}\left(\exp \left(A / l^{2+\varepsilon}\right)-1\right) \sim_{l \rightarrow \infty} \tilde{c}^{\prime} A / l^{2+\varepsilon} .
$$

Combining (A.11) with (A.7) or (A.13) with (A.8), we conclude that, in either case A or case B, there exists a positive constant $C$ such that, for large $l$

$$
\begin{aligned}
\left|\mu_{l}^{*}-\mu_{l}^{*}\right| & \geqq \frac{\lambda_{u}^{-i} C}{l^{2+\varepsilon}}-2 c x_{0}^{*} \lambda_{s}^{l} \geqq C \lambda_{u}^{-l} / l^{2+\varepsilon}-2 c x_{0}^{*} \lambda_{s}^{l} \\
& =\lambda_{u}^{-l}\left(C / l^{2+\varepsilon}-2 c x_{0}^{*}\left(\lambda_{s} \lambda_{u}\right)^{l}\right)>2\left|\Delta_{l}\right| \sim \lambda_{u}^{-2 l} .
\end{aligned}
$$

The last inequality follows from the fact that $\lambda_{s} \lambda_{u}<1(2.2)$ so that $\left(\lambda_{s} \lambda_{u}\right)^{l}$ decreases exponentially with $l$. The proposition is proved.

The last step is to estimate the cardinality for each of the two classes A and B defined respectively by (A.6a) and (A.6b).

Proposition 6. For large $l$, the number of windows in the class $A$ (respectively class $B$ ) is $\sim 2^{\text {hl }}$ (respectively $\sim 2^{h^{\prime} l}$ ) where

$$
h=\left(1+\frac{\log \lambda_{u}}{\log \lambda_{s}^{-1}}\right)^{-1}, \quad h^{\prime}=\left(1+\frac{\log \lambda_{s}^{-1}}{\log \lambda_{u}}\right)^{-1} .
$$

Proof. Equations (A.6a-b) are equivalent to

$$
\begin{aligned}
& i \log \lambda_{u}-(l-j-1) \log \lambda_{s}^{-1}<\log \tilde{c}, \\
& i \log \lambda_{u}-(l-j-1) \log \lambda_{s}^{-1}>\log \tilde{c}^{\prime} .
\end{aligned}
$$

The domains in the $(i, j)$-plane admitted by (A.16) are shown in Fig. 7 as dashed regions A (for class A) and B (for class B). The task, thus, is to consider a subset of simple windows of period $l, W_{A}=\left\{S_{l}\right\}$ (respectively $W_{B}$ ), such that any pair $S_{l}$ and $S_{l}^{\prime}$ in $W_{A}$ (respectively $W_{B}$ ) would have $i$ and $j$ defined by (A.2) belonging to the dashed region A (respectively region B) in Fig. 7. The Proposition 5 then guarantees that they do not overlap, and the cardinality of such a subset will provide with an estimation of the number of windows in the class of type A (respectively type B).

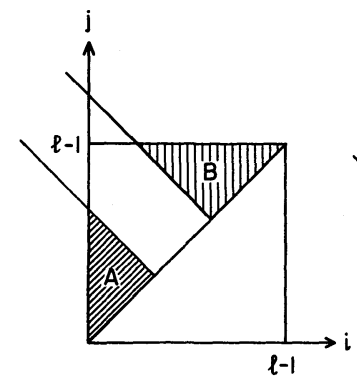

Fig. 7



(III)

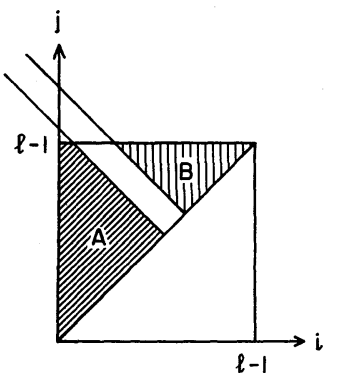

(III) 
As we see from Fig. 7, there are three different cases

$$
\begin{array}{ll}
\tilde{c}<1, & \tilde{c}^{\prime}>1, \\
\tilde{c}<1, & \tilde{c}^{\prime}<1, \\
\tilde{c}>1, & \tilde{c}^{\prime}>1 .
\end{array}
$$

It can be easily checked that $\tilde{c}>1$ and $\tilde{c}^{\prime}<1$ can not be simultaneously realized, given the constraints on $\lambda_{s}$ and $\lambda_{u}(2.2)$. For simplicity let us consider here only the case $I$. The other two cases may be treated in exactly the same way. Look at first the region $A$. It is bounded by

$$
\begin{gathered}
i=0, \text { and } i=j, \\
j=l-1-i \frac{\log \lambda_{u}}{\log \lambda_{s}^{-1}}+\frac{\log \tilde{c}}{\log \lambda_{s}^{-1}} .
\end{gathered}
$$

Let us denote by $i_{\max }$ (respectively $j_{\max }$ ) the intersection of the straight line (A.17b) with $i=j$ (respectively $i=0$ ),

$$
i_{\max }=h\left(l-1+\frac{\log \tilde{c}}{\log \lambda_{s}^{-1}}\right), \quad j_{\max }=i_{\max } / h
$$

with $h$ given in (A.15). Let us choose a fixed string of zeros and ones,

$$
\tilde{\omega}_{\left[j_{\max }\right]+1} \tilde{\omega}_{\left[j_{\max }\right]+2} \ldots \tilde{\omega}_{l-1},
$$

where $[x]$ is the integer part of $x$, and define

$$
\begin{aligned}
& W_{0}=\left\{\left(0 \omega_{1} \omega_{2} \ldots \omega_{\left[j_{\max }\right]} \tilde{\omega}_{\left[j_{\max }\right]+1} \tilde{\omega}_{\left.\left.\left[j_{\max }\right]+2 \ldots \tilde{\omega}_{l-1}\right)\right\},}\right.\right. \\
& W_{1}=\left\{\left(1 \omega_{1} \omega_{2} \ldots \omega_{\left[j_{\max }\right]} \tilde{\omega}_{\left[j_{\max }\right]+1} \tilde{\omega}_{\left.\left.\left[j_{\max }\right]+2 \ldots \tilde{\omega}_{l-1}\right)\right\} .},\right.\right.
\end{aligned}
$$

If $S_{l} \in W_{0}$ and $S_{l}^{\prime} \in W_{1}$, then evidently the values of $i$ and $j$ defined in (A.2) satisfy $i=0, j \leqq\left[j_{\max }\right]$. It follows from the constraint (A.6a) with $i=0$ that

$$
S_{l} \cap S_{l}^{\prime}=\emptyset \text {. }
$$
Likewise, let it be fixed $\tilde{\omega}_{\left[j_{\max }-\alpha\right]+1} \tilde{\omega}_{\left[j_{\max }-\alpha\right]+2} \ldots \tilde{\omega}_{l-1}$, where $\alpha=\frac{\log \lambda_{u}}{\log \lambda_{s}^{-1}}$, and
we define

$$
\begin{aligned}
& W_{00}=\left\{\left(00 \omega_{2} \ldots \omega_{\left[j_{\max }-\alpha\right]} \tilde{\omega}_{\left[j_{\max }-\alpha\right]+1} \tilde{\omega}_{\left.\left.\left[j_{\max }-\alpha\right]+2 \ldots \tilde{\omega}_{l-1}\right)\right\},}\right.\right. \\
& W_{01}=\left\{\left(01 \omega_{2} \ldots \omega_{\left[j_{\max }-\alpha\right]} \tilde{\omega}_{\left[j_{\max }-\alpha\right]+1} \tilde{\omega}_{\left.\left.\left[j_{\max }-\alpha\right]+2 \ldots \tilde{\omega}_{l-1}\right)\right\},} \tilde{\omega}_{10}=\left\{\left(10 \omega_{2} \ldots \omega_{\left[j_{\max }-\alpha\right]} \tilde{\omega}_{\left[j_{\max }-\alpha\right]+1} \tilde{\omega}_{\left.\left.\left[j_{\max }-\alpha\right]+2 \ldots \tilde{\omega}_{l-1}\right)\right\},}\right.\right.\right.\right. \\
& W_{11}=\left\{\left(11 \omega_{2} \ldots \omega_{\left[j_{\max }-\alpha\right]} \tilde{\omega}_{\left[j_{\max }-\alpha\right]+1} \tilde{\omega}_{\left[j_{\max }-\alpha\right]+2} \ldots \tilde{\omega}_{l-1}\right)\right\},
\end{aligned}
$$

then, $W_{00}$ and $W_{01}$, the union of which is included in $W_{0}$, are two nonoverlapping subsets of the simple windows according to the constraint (A.6a) with $i=1$. Idem for $W_{10}$ and $W_{11}$. Combining with the previous statement about $i=0$, we conclude that the four groups of windows defined in (A.21) are not overlapping. Repeating the reasoning in this way, for $i=0,1,2, \ldots,\left[i_{\max }\right]$, we end up by concluding that for any fixed $\tilde{\omega}_{\left[i_{\max }\right]+1} \tilde{\omega}_{\left[i_{\max }\right]+2} \ldots \tilde{\omega}_{l-1}$, all the windows in

$$
W_{A}=\left\{S_{l}=\left(\omega_{0} \omega_{1} \ldots \omega_{\left[i_{\max }\right]} \tilde{\omega}_{\left[i_{\max }\right]+1} \tilde{\omega}_{\left[i_{\max }\right]+2} \ldots \tilde{\omega}_{l-1}\right)\right\}
$$


are not overlapping. The number of such windows are approximately $2^{\left[i_{\max }\right]} \sim 2^{h l}$ with $h$ given in (A.15).

We may proceed with the same consideration for the region B bounded by

By similarly defining $i_{\min }$ as

$$
\begin{gathered}
j=l-1, \quad i=j, \\
j=l-1-i \frac{\log \lambda_{u}}{\log \lambda_{s}^{-1}}+\frac{\log \tilde{c}^{\prime}}{\log \lambda_{s}^{-1}} .
\end{gathered}
$$

$$
i_{\min }=h\left(l-1+\frac{\log \tilde{c}^{\prime}}{\log \lambda_{s}^{-1}}\right)
$$

we may show that for any fixed $\tilde{\omega}_{0} \tilde{\omega}_{1} \ldots \tilde{\omega}_{\left[i_{\min }\right]}$, all the windows in

$$
W_{B}=\left\{S_{l}=\left(\tilde{\omega}_{0} \tilde{\omega}_{1} \ldots \tilde{\omega}_{\left[i_{\text {min }}\right]} \omega_{\left[i_{\text {min }}\right]+1} \omega_{\left[i_{\text {min }}\right]+2} \ldots \omega_{l-1}\right)\right\}
$$

are not overlapping. The number of such windows is approximately $2^{l-1-\left[i_{\min }\right]} \sim 2^{l h^{\prime}}$, where $h^{\prime}$ is given in (A.15).

Thus, we have constructed two classes of windows, $W_{A}$ and $W_{B}$. Within each of them, any pair of windows do not overlap. Of course, these two classes may overlap one with the other. For our purpose, we may select the first class $W_{A}$ which is larger than the second one, since $\lambda_{s}^{-1}>\lambda_{u}$ implies that $h>h^{\prime}$. We also note that this class is not unique; in fact any choice of the subsequence of string $\tilde{\omega}_{\left[i_{\max }\right]+1} \tilde{\omega}_{\left[i_{\max }\right]+2} \ldots \tilde{\omega}_{l-1}$ will do in our construction of $W_{A}$. However, the largest class of nonoverlapping windows we were able to construct has the number as stated in Lemma 2.

Acknowledgements. It is a great pleasure to thank Prof. G. Nicolis for his support on the present research; and Profs. I. Prigogine and L. Reichl for the hospitality at the University of Texas at Austin. Discussions with Dr. P. Gaspard are warmly acknowledged. I wish to express my sincere gratitude to Prof. C. Robinson for an important correspondence. Thanks are also due to the gentleman who has suggested Fig. 1, and whose name is unfortunately unknown to me.

\section{References}

1. Billingsley, P.: Ergodic theory and information. Huntington: Krieger 1978

2. Chenciner, A., Iooss, G.: Arch. Rat. Mech. Anal. 69, 109-198 (1979)

3. Gambaudo, J.M., Tresser, C.: J. Stat. Phys. 32, 455-476 (1983)

4. Gaspard, P., Wang, X.-J.: J. Stat. Phys. 48, 151-199 (1987)

5. Gavrilov, N.K., Shil'nikov, L.P.: Math. USSR Sbornik 17, 467-485 (1972); 19, 139-156 (1973)

6. Guckenheimer, J., Holmes, Ph.J.: Nonlinear oscillations, dynamical systems, and bifurcations of vector fields. Berlin, Heidelberg, New York: Springer 1983

7. Newhouse, S.: Topology 12, 9-18 (1974)

8. Newhouse, S.: Publ. Math. I.H.E.S. 50, 101-151 (1979)

9. Oxtoby, J.C.: Measure and category. Berlin, Heidelberg, New York: Springer 1980

10. Robinson, C.: Commun. Math. Phys. 90, 433-459 (1983)

11. Smale, S.: Bull. Am. Math. Soc. 73, 747-817 (1967)

12. Tedeschini-Lalli, L., Yorke, J.A.: Commun. Math. Phys. 106, 635-657 (1986)

13. Wang, X.-J.: Ph. D. Dissertation, University of Brussels (1987)

Communicated by J.-P. Eckmann

Received February 27, 1989; in revised form February 16, 1990 